\title{
Characterization Sets for the Nucleolus in Balanced Games
}

\author{
Tamás Solymosia ${ }^{a}$ Balázs Sziklai ${ }^{a, b, *}$ \\ ${ }^{a}$ Corvinus University of Budapest, Department of Operations Research and Actuarial Sciences, H-1093 Budapest Fốvám \\ tér 8., Email: tamas.solymosi@uni-corvinus.hu \\ b 'Momentum' Game Theory Research Group, Centre for Economic and Regional Studies, Hungarian Academy of \\ Sciences. H-1112 Budapest Budaörsi út 45., Email: sziklai.balazs@krtk.mta.hu
}

\begin{abstract}
We provide a new modus operandi for the computation of the nucleolus in cooperative games with transferable utility. Using the concept of dual game we extend the theory of characterization sets. Dually essential and - if the game is monotonic - dually saturated coalitions determine both the core and the nucleolus whenever the core is non-empty. We show how these two sets are related to the existing characterization sets. In particular we prove that if the grand coalition is vital then the intersection of essential and dually essential coalitions forms a characterization set itself.
\end{abstract}

Keywords: Cooperative game theory; Core; Nucleolus; Characterization sets; Dual game

\section{Introduction}

The nucleolus, introduced by Schmeidler [29], is one of the most frequently applied cooperative game theoretical solution concept. Much like the Shapley-value it suffers from computational difficulties. Determining the nucleolus is $\mathcal{N} \mathcal{P}$-hard for various classes of games such as minimum cost spanning tree games [7, voting games [6] and flow and linear production games [5]. Recently Greco et al. [13] provided a non-trivial upper bound for its complexity. On the other hand there are known polynomial time algorithms for computing the nucleolus of important families of cooperative games, like standard tree [21], assignment [31], matching [16] and bankruptcy games [2]. In addition, Kuipers [18] and Arin and Inarra [1] developed methods to compute the nucleolus for convex games.

In contrast to the Shapley-value, there is no formula for the nucleolus. One way to compute it is to use linear programming. The sequential LP approach of Maschler et al. [20] was the first computationally tractable one. Since then there have been many attempts to improve the computation process see e.g. [28, 8, 25, 26]. Although all

\footnotetext{
${ }^{*}$ Corresponding author
}

the proposed LPs consist of exponentially many inequalities they can be solved efficiently if one knows which constraints are redundant. Huberman [15], Granot et al. [12] and Reijnierse and Potters [27] provided methods to identify coalitions that correspond to non-redundant constraints.

This idea was already suggested by Megiddo [23], but the formal theoretical framework was developed by Granot et al. [12. They introduced the concept of characterization sets, namely a collection of coalitions that itself determines the nucleolus, and proved that if the size of the characterization set is polynomially bounded in the number of players, then the nucleolus of the game can be computed in strongly polynomial time. A collection that characterizes the nucleolus in a game need not characterize it in another game. Thus we are interested in a property of the coalitional function that describes a characterization set in every game in a sufficiently large class of games. Huberman [15] was the first to show that such a property exists in the very important class of balanced games. He introduced the concept of essential coalitions which are coalitions that have no weakly majorizing partition, and proved that if the core is non-empty, the family of essential coalitions itself determines 
the nucleolus. Granot et al. 12] provided another collection that characterize the nucleolus in cost games with non-empty core.

We introduce two new characterization sets: dually essential and dually saturated coalitions. We show that both sets (in themselves) determine the core, and if the core is non-empty they determine the nucleolus as well. We conclude by analyzing the relationship of the four known characterizing properties.

\section{Game-theoretic framework}

A cooperative game with transferable utility is an ordered pair $(N, v)$ consisting of the player set $N=\{1,2, \ldots, n\}$ and a characteristic function $v$ : $2^{N} \rightarrow \mathbb{R}$ with $v(\emptyset)=0$. The value $v(S)$ represents the worth of coalition $S$. Let $\mathcal{P}=2^{N} \backslash\{\emptyset, N\}$ denote the family of the non-trivial coalitions. A cooperative game $(N, v)$ is called monotonic if $S \subseteq$ $T \subseteq N \Rightarrow v(S) \leq v(T)$. A general assumption in cooperative games is that the grand coalition $(N)$ forms. The question is then how to distribute $v(N)$ among the players in some fair way.

An outcome for a cooperative game $\Gamma=(N, v)$ is a vector $x \in \mathbb{R}^{N}$ that represents the payoff of each player. For convenience, we introduce the notation $x(S)=\sum_{i \in S} x_{i}$ for any $S \subseteq N$, and instead of $x(\{i\})$ we simply write $x(i)$. A solution is called efficient if $x(N)=v(N)$ and individually rational if $x(i) \geq v(i)$ for all $i \in N$. Efficient solutions will also be called allocations. The imputation set $\mathbf{I}(\Gamma)$ of the game $\Gamma$ consists of the efficient and individually rational solutions, formally, $\mathbf{I}(\Gamma)=\{x \in$ $\mathbb{R}^{N} \mid x(N)=v(N), x(i) \geq v(i)$ for all $\left.i \in N\right\}$. Given a payoff vector $x \in \mathbb{R}^{N}$, we define the satisfaction of coalition $S$ in game $\Gamma$ as

$$
\operatorname{sat}_{\Gamma}(S, x):=x(S)-v(S) .
$$

The satisfaction of the grand coalition is clearly zero at any allocation. The core $\mathbf{C}(\Gamma)$ of cooperative game $\Gamma$ is the set of allocations where all the satisfaction values are non-negative. Formally,

$$
\begin{aligned}
\mathbf{C}(\Gamma)=\left\{x \in \mathbb{R}^{N}\right. & \mid \operatorname{sat}_{\Gamma}(N, x)=0, \\
& \left.\operatorname{sat}_{\Gamma}(S, x) \geq 0 \text { for all } S \in \mathcal{P}\right\} .
\end{aligned}
$$

A game is called balanced if its core in non-empty. In this paper we will consider only balanced games. Notice that core vectors of monotonic games are non-negative. Indeed, $x(i) \geq v(i) \geq v(\emptyset)=0$ for all $i \in N$.

We say that a vector $x \in \mathbb{R}^{m}$ lexicographically precedes $y \in \mathbb{R}^{m}$ (denoted by $x \preceq y$ ) if either $x=y$ or there exists a number $1 \leq j \leq m$ such that $x_{i}=y_{i}$ if $i<j$ and $x_{j}<y_{j}$. Let $\Gamma=(N, v)$ be a game and let $\theta^{\mathcal{P}}(x) \in \mathbb{R}^{2^{n}-2}$ be the vector that contains the $2^{n}-2$ satisfaction values $\operatorname{sat}_{\Gamma}(S, x)$, $S \in \mathcal{P}$, in a non-decreasing order.

Definition 1. The nucleolus of a cooperative game $\Gamma$ is the subset of the payoff vectors $x \in \boldsymbol{I}(\Gamma)$ that lexicographically maximize $\theta^{\mathcal{P}}(x)$. Formally,

$$
\mathbf{N}(\Gamma)=\left\{x \in \boldsymbol{I}(\Gamma) \mid \theta^{\mathcal{P}}(y) \preceq \theta^{\mathcal{P}}(x) \forall y \in \boldsymbol{I}(\Gamma)\right\} .
$$

Schmeidler 29] proved that $\mathbf{N}(\Gamma)$ consists of a single point, and that it is a continuous function of the characteristic function. Although formally a set, we will consider $N(\Gamma)$ as an allocation vector. It is straightforward that $\mathbf{N}(\Gamma) \in \mathbf{C}(\Gamma)$ whenever $\mathbf{C}(\Gamma)$ is non-empty.

Kohlberg [17] offered a method to verify whether an allocation coincides with the nucleolus. Let $e_{S} \in\{0,1\}^{N}$ denote the membership vector of coalition $S$, that is, $\left(e_{S}\right)_{i}=1$ if $i \in S$ and $\left(e_{S}\right)_{i}=0$ otherwise. A collection of coalitions $\mathcal{B}_{S} \subseteq \mathcal{P}$ is said to be $S$-balanced if there exist positive weights $\lambda_{T}, T \in \mathcal{B}_{S}$, such that $\sum_{T \in \mathcal{B}_{S}} \lambda_{T} e_{T}=e_{S}$. An $N-$ balanced collection is simply called balanced.

Theorem 2 (Kohlberg [17]). Let $\Gamma=(N, v)$ be a game. Then an allocation $x$ is the nucleolus if and only if for all $y \in \mathbb{R}$ the collection $\{S \in$ $\left.\mathcal{P} \mid \operatorname{sat}_{\Gamma}(S, x) \leq y\right\}$ is balanced or empty.

\section{Characterization sets}

In the following we will use the formalism of Granot et al. [12.

Definition 3. Let $\Gamma^{\mathcal{F}}=(N, \mathcal{F}, v)$ be a cooperative game with coalition formation restrictions, where $\emptyset \neq \mathcal{F} \subseteq \mathcal{P}$ consists of all coalitions deemed permissible besides the grand coalition $N$. Let $\theta^{\mathcal{F}}(x) \in$ 
$\mathbb{R}^{|\mathcal{F}|}$ be the restricted vector that contains the satisfaction values $\operatorname{sat}_{\Gamma}(S, x), S \in \mathcal{F}$ in a non-decreasing order. Furthermore, let $\mathbf{N}\left(\Gamma^{\mathcal{F}}\right)$ be defined as the set of allocations that lexicographically maximize $\theta^{\mathcal{F}}(x)$. Then $\mathcal{F}$ is called a characterization set for the nucleolus of the game $\Gamma=(N, v)$, if $\mathbf{N}\left(\Gamma^{\mathcal{F}}\right)=$ $\mathbf{N}(\Gamma)$.

Note the different roles of $N$ and the coalitions in $\mathcal{F}$ in the above optimization. The satisfaction of the grand coalition is required to be constantly zero (defining the feasible set), whereas the satisfactions of the smaller permissible coalitions form the objective function. Notice that $\mathbf{N}\left(\Gamma^{\mathcal{F}}\right)$ is a singleton if and only if $\operatorname{rank}\left(\left\{e_{S}: S \in\{N\} \cup \mathcal{F}\right\}\right)=n$. Most relevant to our setting is the following theorem of Granot et al. [12.

Theorem 4. Let $\Gamma$ be a cooperative game with a non-empty core. The non-empty collection $\mathcal{F} \subseteq$ $\mathcal{P}$ is a characterisation set for the nucleolus of $\Gamma$ if for every $S \in \mathcal{P} \backslash \mathcal{F}$ there exists a non-empty subcollection $\mathcal{F}_{S}$ of $\mathcal{F}$, such that

i. $\operatorname{sat}_{\Gamma}(T, x) \leq \operatorname{sat}_{\Gamma}(S, x)$ for all $x \in \mathbf{C}(\Gamma)$, whenever $T \in \mathcal{F}_{S}$,

ii. $e_{S}$ can be expressed as a linear combination of the vectors in $\left\{e_{T}: T \in \mathcal{F}_{S} \cup\{N\}\right\}$.

Observe that the above conditions are sufficient but not at all necessary. Take for example the (superadditive and balanced) profit game with four players $N=\{1,2,3,4\}$ and the following coalitional function: $v(i)=0, v(i, j)=1, v(i, j, k)=3$ for any $i, j, k \in N$ and $v(N)=4$. Then the 2player coalitions and the grand coalition are sufficient to determine the nucleolus, which is given by $z(i)=1$ for all $i \in N$. However, the 3 -player coalitions have smaller satisfaction values at $z$, thus the first condition of Theorem 4 is violated. Notice that in this game the 3-player coalitions and the grand coalition are also sufficient to determine the nucleolus.

In general neither the 2-player nor the 3-player coalitions (and the grand coalition) characterize the nucleolus. The fact that in this example they did was due to the particular choice (most importantly the symmetry) of the coalitional function.
We would like to identify properties of coalitions that characterize the nucleolus independently of the realization of the coalitional function.

The Kohlberg-criterion applied to games with coalition formation restrictions yields the following theorem.

Theorem 5 (Maschler et al. [22]). Let $\mathcal{F}$ be a characterization set and $x$ be an imputation of the game $\Gamma$ with $\mathbf{C}(\Gamma) \neq \emptyset$. Then $x=\mathbf{N}(\Gamma)$ if and only if for all $y \in \mathbb{R}$ the collection $\left\{S \in \mathcal{F} \mid \operatorname{sat}_{\Gamma}(S, x) \leq\right.$ $y\}$ is balanced or empty.

A similar criterion appears in [14]. With the help of the Kohlberg-criterion the problem of finding the nucleolus is reduced to finding the right characterization set. The first characterization set for balanced games is due to Huberman [15].

Definition 6 (Essential coalitions). Let $N$ be a set of players, $(N, v)$ a cooperative game. Coalition $S \in \mathcal{P}$ is called essential in game $\Gamma=(N, v)$ if it can not be partitioned as $S=S_{1} \dot{\cup} \ldots \dot{\cup} S_{k}$ with $k \geq 2$ and $S_{j} \neq \emptyset$ for all $1 \leq j \leq k$ such that $S$ is weakly majorized by $S_{1}, \ldots, S_{k}$, that is

$$
v(S) \leq v\left(S_{1}\right)+\ldots+v\left(S_{k}\right) .
$$

The set of essential coalitions is denoted by $\mathcal{E}(\Gamma)$. A coalition that is not essential is called inessential.

A similar criterion can be formulated for cost games, but there the inequality is reversed, i.e. $c(S) \geq c\left(S_{1}\right)+\ldots+c\left(S_{k}\right)$ must hold.

Notice that we call only a non-trivial coalition essential, although $\emptyset$ would always qualify since it can not be partitioned at all, and $N$ could also qualify in certain games. On the other hand, the singleton coalitions are always essential in every game.

It is easily seen that each inessential coalition has a weakly majorizing partition which consists exclusively of essential coalitions. Moreover, the core is determined by the efficiency equation $\operatorname{sat}_{\Gamma}(N, x)=0$ and the $\operatorname{sat}_{\Gamma}(S, x) \geq 0$ inequalities corresponding to the essential coalitions, all the other inequalities can be discarded from the core system. The following important result is due to Huberman 15 . 
Theorem 7 (Huberman [15]). If the core of the game is non-empty then the essential coalitions form a characterization set for the nucleolus.

The theorem means that in a balanced game the essential coalitions and the grand coalition are sufficient to determine the nucleolus. This observation helps us to eliminate large coalitions which are redundant for the nucleolus. To detect small coalitions that are unnecessary for the nucleolus, we need the concept of dual game.

Definition 8. The dual game $\Gamma^{*}=\left(N, v^{*}\right)$ of game $\Gamma=(N, v)$ is defined by the coalitional function $v^{*}(S):=v(N)-v(N \backslash S)$ for all $S \subseteq N$.

Notice that $v^{*}(\emptyset)=0, v^{*}(N)=v(N)$, and $\left(v^{*}\right)^{*}(S)=v(S)$ for all $S \subseteq N$. It will be useful to think of the dual game of a profit game as a cost game and vice versa. It can be easily checked that $v$ is monotonic if and only if $v^{*}$ is monotonic, and $v$ is supermodular if and only if $v^{*}$ is submodular. However, there is no such dualization relation between superadditivity and subadditivity. Oishi and Nakayama [24] provide examples, how important classes of cooperative games are connected through the dual game relation.

We can identify small redundant coalitions, if we apply Huberman's argument to the dual game.

Definition 9 (Dually essential coalitions). Let $N$ be a set of players, $(N, v)$ a cooperative game. Coalition $S \in \mathcal{P}$ is called dually essential in game $(N, v)$ if its complement can not be partitioned as $N \backslash S=$ $\left(N \backslash T_{1}\right) \dot{\cup} \ldots \dot{\cup}\left(N \backslash T_{k}\right)$ with $k \geq 2$ and $T_{j} \neq N$ for all $1 \leq j \leq k$ such that

$$
v^{*}(N \backslash S) \geq v^{*}\left(N \backslash T_{1}\right)+\ldots+v^{*}\left(N \backslash T_{k}\right),
$$

or equivalently,

$$
v(S) \leq v\left(T_{1}\right)+\ldots+v\left(T_{k}\right)-(k-1) v(N) .
$$

The set of dually essential coalitions is denoted by $\mathcal{D E}(\Gamma)$. A coalition that is not dually essential is called dually inessential.

Similarly, when we formulate this requirement for cost games, the inequality gets reversed, i.e. $c(S) \geq c\left(T_{1}\right)+\ldots+c\left(T_{k}\right)-(k-1) c(N)$ must hold for such coalitions.
Notice that we call only a non-trivial coalition dually essential, although the grand coalition would always, and the empty coalition could sometimes qualify. On the other hand, all $(n-1)$-player coalitions are dually essential in any game. Observe that $S \in \mathcal{P}$ is dually essential if and only if $N \backslash S \in \mathcal{P}$ is essential in the dual game.

If $S \in \mathcal{P}$ is dually inessential then it is contained in each of the coalitions $T_{1}, \ldots, T_{k} \in \mathcal{P}$ in the above expression, but every player in $N \backslash S$ appears exactly $k-1$ times in this family. We call such a system of coalitions an overlapping decomposition of $S$. For a more general definition, where the complements of the overlapping coalitions need not form a partition of the complement coalition, see e.g. Brânzei et al. [3] and the references therein.

It is clear that if $S, T \in \mathcal{P}$ are dually inessential coalitions and $T$ appears in an overlapping decomposition of $S$, then $S \subsetneq T$ so $S$ cannot appear in an overlapping decomposition of $T$. Consequently, in a cooperative game each dually inessential coalition has a weakly majorizing overlapping decomposition which consists exclusively of dually essential coalitions. Moreover, the core of $\Gamma$ is also determined by the dual efficiency equation $\operatorname{sat}_{\Gamma^{*}}(N, x)=0$ and the $\operatorname{sat}_{\Gamma^{*}}(S, x) \geq 0$ dual inequalities corresponding to the complements of the dually essential coalitions, all the other dual inequalities can be discarded from the dual core system. The main feature of dually essential coalitions is that together with the grand coalition they are sufficient to determine the nucleolus in balanced games. The next theorem is the dual counterpart of Theorem 7

Proposition 10. If $\mathbf{C}(\Gamma) \neq \emptyset$, then the dually essential coalitions form a characterization set for $\mathbf{N}(\Gamma)$.

Proof. There are various ways to derive this result. A formal proof can be obtained by applying the arguments of Huberman [15] to the dual game. Here we pursue another way and deduce it from Theorem 4 .

Let $S \in \mathcal{P}$ be a dually inessential coalition in the balanced profit game $\Gamma=(N, v)$. As remarked earlier, $S$ has a weakly majorizing overlapping de- 
composition $T_{1}, \ldots, T_{k}(k \geq 2)$ which consists exclusively of dually essential coalitions. Hence ii. of Theorem 4 follows immediately. To see condition i., let $x \in \mathbf{C}(\Gamma)$. Then

$$
\begin{aligned}
& v(S) \leq v\left(T_{1}\right)+\ldots+v\left(T_{k}\right)-(k-1) v(N) \\
& v(S)-x(S) \leq v\left(T_{1}\right)+\ldots+v\left(T_{k}\right)- \\
&-(k-1) x(N)-x(S) \\
&-\operatorname{sat}_{\Gamma}(S, x) \leq-\left(\operatorname{sat}_{\Gamma}\left(T_{1}, x\right)+\ldots+\operatorname{sat}_{\Gamma}\left(T_{k}, x\right)\right) \\
& \operatorname{sat}_{\Gamma}(S, x) \geq \operatorname{sat}_{\Gamma}\left(T_{1}, x\right)+\ldots+\operatorname{sat}_{\Gamma}\left(T_{k}, x\right) \\
& \operatorname{sat}_{\Gamma}(S, x) \geq \operatorname{sat}_{\Gamma}\left(T_{j}, x\right) \geq 0 \quad \forall j=1, \ldots, k,
\end{aligned}
$$

where the second inequality comes from $v(N)=$ $x(N)$, while the third from the identity $x\left(T_{1}\right)+\ldots+$ $x\left(T_{k}\right)=(k-1) x(N)+x(S)$ implied by $N \backslash S=$ $\left(N \backslash T_{1}\right) \cup \dot{\cup}\left(N \backslash T_{k}\right)$, and the last one from the non-negativity of the satisfaction values at any core allocation.

If we look for an as small characterisation set as possible for balanced games, in light of Theorems 7 and 10 it seems natural to eliminate both inessential coalitions and dually inessential coalitions at the same time. However, the intersection of essential and dually essential coalitions need not yield a characterization set. For example, in additive games only the singleton coalitions are essential, and only the $(n-1)$-player coalitions are dually essential.

The problem lies in what we call a cycle in the decomposition. This occurs when coalition $S$ can be discarded because of a collection that contains coalition $T$, and $T$ can also be discarded because of a collection that contains $S$ or another coalition $R$ that can be eliminated because of a collection that contains $S$, etc. As remarked after both definitions, such a cycle in the decomposition cannot occur if we apply only essentiality or only dual essentiality. Strongly essential coalitions discussed by Brânzei et al. [3] are not immune to this kind of failure, hence they might not form a characterization set for the nucleolus in a balanced game in which the grand coalition is not vital.

Before we show when the two types of concepts can be mixed, we need some preparation.
A coalition $S$ is called vital if for any $S$-balanced collection $\mathcal{B}_{S}$ and any system $\left(\lambda_{T}\right)_{T \in \mathcal{B}_{S}}$ of balancing weights for $\mathcal{B}_{S}, \sum_{T \in \mathcal{B}_{S}} \lambda_{T} v(T)<v(S)$ (see [9. 30]). It is easily seen that the family of vital coalitions (just like essential coalitions) and the grand coalition are sufficient to determine the core. However, Maschler et al. 20] demonstrated that vital coalitions (unlike essential coalitions) and the grand coalition do not necessarily characterize the nucleolus in a balanced game.

The next theorem provides a sufficient condition for $\mathcal{E}(\Gamma) \cap \mathcal{D E}(\Gamma)$ to be a characterization set for the nucleolus in a balanced game.

Theorem 11. Let $\Gamma=(N, v)$ be a game with a non-empty core. If the grand coalition is vital, the collection $\mathcal{E}(\Gamma) \cap \mathcal{D E}(\Gamma)$ forms a characterization set of $\mathbf{N}(\Gamma)$.

Proof. Suppose coalition $S$ is redundant, that is, either inessential or dually inessential (or both). Then there is a series of coalitions $S_{1}, \ldots, S_{k}$ in $\mathcal{E}(\Gamma)$ or in $\mathcal{D E}(\Gamma)$ that have smaller satisfaction value than $S$ and whose membership vectors span $e_{S}$. Lexicographically maximizing the satisfactions of $S_{1}, \ldots, S_{k}$ the satisfaction of $S$ becomes fixed. If there is no cycle in the decomposition then there exists a decomposition of $S_{i}$ for $i=1, \ldots, k$ that consist entirely of coalitions $T_{1}^{i}, \ldots, T_{r_{i}}^{i}$ that belong to $\mathcal{E}(\Gamma) \cap \mathcal{D} \mathcal{E}(\Gamma)$. Lexicographically maximizing the satisfactions of $T_{1}^{i}, \ldots, T_{r_{i}}^{i}$ the satisfaction of $S_{i}$ becomes fixed. Thus indirectly the satisfaction of $S$ becomes fixed. We conclude that, if there is no cycle in the decomposition, then by Theorem 4 $\mathcal{E}(\Gamma) \cap \mathcal{D E}(\Gamma)$ is a characterization set.

By contradiction suppose that the grand coalition is vital, but there is a cycle $T_{1}, T_{2}, \ldots, T_{r}$ in the decomposition. Note that we may assume without loss of generality that the series alternates between inessentiality and dual inessentiality. If $T_{\ell}$ and $T_{\ell+1}$ were deemed redundant for the same reason (e.g. they are both inessential) then the inequality that shows inessentiality of $T_{\ell}$ can be refined by the inequality that shows inessentiality of $T_{\ell+1}$. Let us assume that $T_{1}$ is inessential - the proof is the same if $T_{1}$ is dually inessential. Thus, using the definition of essentiality and dual essen- 
tiality

$$
\begin{aligned}
v\left(T_{1}\right) & \leq v\left(T_{2}\right)+\sum_{j=1}^{k_{1}} v\left(S_{j}^{1}\right) \\
v\left(T_{2}\right) & \leq v\left(T_{3}\right)+\sum_{j=1}^{k_{2}} v\left(S_{j}^{2}\right)-k_{2} \cdot v(N) \\
v\left(T_{3}\right) & \leq v\left(T_{4}\right)+\sum_{j=1}^{k_{3}} v\left(S_{j}^{3}\right) \\
\vdots & \\
v\left(T_{r}\right) & \leq v\left(T_{1}\right)+\sum_{j=1}^{k_{r}} v\left(S_{j}^{r}\right)-k_{r} \cdot v(N)
\end{aligned}
$$

In words $T_{1}$ is inessential because of the collection $T_{2}, S_{1}^{1}, \ldots, S_{k_{1}}^{1}$ (these are all essential coalitions, thus the inequality cannot be refined any more). Then $T_{2}$ is dually inessential because of the collection $T_{3}, S_{1}^{2}, \ldots, S_{k_{2}}^{2}$ compose an overlapping decomposition of $T_{2}$ (and these are all dually essential). And so on until finally $T_{r}$ is deemed redundant because of $T_{1}, S_{1}^{r}, \ldots, S_{k_{r}}^{r}$. Note that there may be coalitions among $S_{1}^{1}, \ldots, S_{1}^{2}, \ldots, S_{1}^{r}, \ldots, S_{k_{r}}^{r}$ that coincide. Using indicator functions and the conditions of inessentiality and dual inessentiality, we get

$$
\begin{gathered}
e_{T_{1}}=e_{T_{2}}+\sum_{j=1}^{k_{1}} e_{S_{j}^{1}} \\
e_{T_{2}}=e_{T_{3}}+\sum_{j=1}^{k_{2}} e_{S_{j}^{2}}-k_{2} \cdot e_{N} \\
e_{T_{3}}=e_{T_{4}}+\sum_{j=1}^{k_{3}} e_{S_{j}^{3}} \\
\vdots \\
e_{T_{r}}=e_{T_{1}}+\sum_{j=1}^{k_{r}} e_{S_{j}^{r}}-k_{r} \cdot e_{N}
\end{gathered}
$$

Thus by summing (1)-(4) we obtain that

$$
v(N) \leq \frac{1}{k_{2}+k_{4}+\cdots+k_{r}} \sum_{i=1}^{r} \sum_{j=1}^{k_{i}} v\left(S_{j}^{i}\right)
$$

while from (5)-(8) we gather that

$$
e_{N}=\frac{1}{k_{2}+k_{4}+\cdots+k_{r}} \sum_{i=1}^{r} \sum_{j=1}^{k_{i}} e_{S_{j}^{i}}
$$

i.e. the collection $S_{1}^{1}, \ldots, S_{1}^{2}, \ldots, S_{1}^{r}, \ldots, S_{k_{r}}^{r}$ is balanced. This contradicts the fact that the grand coalition is vital.

\section{Monotonic balanced games}

Granot et al. 12, proposed a characterization set for cost games. Informally, a coalition is called saturated in a cost game if every new member would increase the cost of the coalition (or putting it differently, the coalition contains all the players that could join for free). The grand coalition and the empty set are not considered saturated, although $N$ would always $\emptyset$ would sometimes qualify. They proved that the saturated coalitions together with the $(n-1)$-player coalitions, $\mathcal{S}(\Gamma)$, form a characterization set in balanced monotonic cost games. In fact Granot et al. [12] proved a slightly stronger statement, namely, that the intersection of saturated and essential coalitions forms a characterization set in this class of games.

Similarly to the other characterization sets, $\mathcal{S}(\Gamma)$ also induces a representation of the core $\mathbf{C}(\Gamma)$. Let us mention here that just because a collection of coalitions determines the core it does not necessarily characterize the nucleolus of the game. Maschler et al. 20] presented two games with the same core, but with different nucleoli.

We now convert the concept of saturatedness to monotonic profit games based on the dualization correspondence between profit and cost games.

Definition 12 (Dually saturated coalitions). Let $(N, v)$ be a monotonic profit game and $S \in \mathcal{P}$ be an arbitrary non-trivial coalition. We say that $S$ is dually saturated if $v(S \backslash i)<v(S)$ for any $i \in S$.

In other words every member contributes to the worth of coalition $S$. Notice that although $\emptyset$ vacuously always satisfies this property we do not call it a dually saturated coalition. Similarly, also $N$ is not considered saturated even if it would qualify in certain games. Observe that $S \in \mathcal{P}$ is saturated in the monotonic cost game $c$ if and only if $N \backslash S \in \mathcal{P}$ is dually saturated in the monotonic profit game $c^{*}$. Let $\mathcal{D S}^{+}(\Gamma)$ denote the set of all dually saturated coalitions and $\mathcal{D S}(\Gamma)=\mathcal{D S}^{+}(\Gamma) \cup\{i \mid i \in$ $N\}$. 
The following definition is needed for our next theorem. Let $(N, v)$ be a monotonic game and $S \in$ $\mathcal{P}$ a dually non-saturated coalition, then we say that $\underline{S} \neq \emptyset$ is a lower closure of $S$ if $\underline{S} \subset S, v(\underline{S})=$ $v(S)$ and $\underline{S}$ is a dually saturated coalition. Note that if $S$ has no lower closure, then no member contributes to the worth of $S$ or to any subset of $S$. Hence $v(S)=v(i)=v(\emptyset)=0$ for any $i \in S$.

Proposition 13. Let $\Gamma=(N, v)$ be a monotonic game with a non-empty core, then $\mathcal{D S}(\Gamma)$ forms a characterization set for $\mathbf{N}(\Gamma)$.

Proof. Again we will use Theorem 4 . Let $S \in \mathcal{P}$ be a dually non-saturated coalition. If $S$ has no lower closure then $v(S)=v(i)=0$ for any $i \in S$. From this observation it also follows that $\operatorname{sat}_{\Gamma}(\{i\}, x) \leq$ $\operatorname{sat}_{\Gamma}(S, x)$ for any $i \in S$ and for any allocation $x$. Since all the singleton coalitions are included in $\mathcal{D S}(\Gamma)$ by Theorem 4, $S$ can be discarded. Finally let $\underline{S}$ be a lower closure of $S$ and let $S \backslash \underline{S}=T$, then

$$
\begin{aligned}
\operatorname{sat}_{\Gamma}(\underline{S}, x)+x(T)= & x(\underline{S})+x(T)-v(\underline{S})= \\
& =x(S)-v(S)=\operatorname{sat}_{\Gamma}(S, x) .
\end{aligned}
$$

Since core vectors are non-negative this also means $\operatorname{sat}_{\Gamma}(\underline{S}, x) \leq \operatorname{sat}_{\Gamma}(S, x)$ for any $x \in \mathbf{C}(\Gamma)$. Now we show that $\operatorname{sat}_{\Gamma}(\{i\}, x) \leq \operatorname{sat}_{\Gamma}(S, x)$ for any $i \in T$.

$$
\begin{gathered}
\operatorname{sat}_{\Gamma}(\{i\}, x)=x(i)-v(i) \leq x(i)= \\
x(S)-x(S \backslash i)+v(S \backslash i)-v(S)= \\
\operatorname{sat}_{\Gamma}(S, x)-\operatorname{sat}_{\Gamma}(S \backslash i, x) \leq \operatorname{sat}_{\Gamma}(S, x)
\end{gathered}
$$

We have shown that for any $S \in \mathcal{P} \backslash \mathcal{D S}(\Gamma)$ there exist a subcollection $\mathcal{F}$ of $\mathcal{D} \mathcal{S}(\Gamma)$, such that $\mathcal{F}$ fulfills both conditions of Theorem 4. Hence $\mathcal{D S}(\Gamma)$ is a characterization set for $\mathbf{N}(\Gamma)$.

Next we show a relationship between essential and dually saturated coalitions.

Proposition 14. Let $\Gamma=(N, v)$ be a monotonic profit game, then $\mathcal{E}(\Gamma) \subseteq \mathcal{D} \mathcal{S}(\Gamma)$.
Proof. The singleton coalitions are all members of both $\mathcal{E}(\Gamma)$ and $\mathcal{D S}(\Gamma)$. Let $S$ be a dually nonsaturated coalition such that $|S|>1$. Then there exists $i \in S$ such that $v(S)=v(S \backslash i)$. By monotonicity $v(i) \geq 0$, hence $v(S) \leq v(S \backslash i)+v(i)$. Thus $S$ is inessential.

There is a symmetrical result for dually essential and saturated coalitions. It can be shown that if $\Gamma=(N, c)$ is a monotonic cost game, then $\mathcal{D} \mathcal{E}(\Gamma) \subseteq \mathcal{S}(\Gamma)$.

Let us say a few words about the applicability of the above results. Sziklai [33. provides an example where the dually essential coalitions help to find the nucleolus of directed acyclic graph games. Dually essential coalitions seem to be more useful than saturated coalitions since they define a smaller characterization set, which in turn implies a smaller LP. However usually it is also harder to determine whether a coalition is dually essential or not. Saturatedness on the other hand can be checked easily. For instance for airport games [19] there exist at most $n$ saturated coalitions, which can be easily determined from the characteristic function. In case of bankruptcy games there can be exponential many essential and dually essential coalitions, however the size of their intersection is always linear. In such games the grand coalition is vital, hence Theorem 11 can be applied [32]. This example is not an exception, but rather the general case: balanced games with a vital grand coalition represent almost all the class of balanced games (see [10), thus Theorem 11 can be very useful in practice.

Finally let us stress that the four characterization sets that we analyzed in this paper can only help when the game in question has a non-empty core. Göthe-Lundgren et al. [11] computed the nucleolus of a vehicle routing game using the concept of essential coalitions. However as Chardaire [4] pointed out these games are not necessarily balanced, hence their approach is flawed.

\section{Acknowledgements}

The authors would like to thank Prof. Tamás Kis of Institute for Computer Science and Con- 
trol (MTA SZTAKI) and the anonymous referees of the CoopMAS-2015 conference for their valuable comments. Research was supported by OTKA grants K101224 and K108383 and by the Hungarian Academy of Sciences Momentum Programme Strategic Interactions Research Group (Solymosi) and Game Theory Research Group (Sziklai, LD004/2010). This research was partially supported by Pallas Athene Domus Scientiae Foundation. The views expressed are those of the authors' and do not necessarily reflect the official opinion of Pallas Athene Domus Scientiae Foundation.

[1] Arin, J. and Inarra, E. (1998). A characterization of the nucleolus for convex games. Games and Economic Behaviour, 23:12-24.

[2] Aumann, R. and Maschler, M. (1985). Game theoretic analysis of a bankruptcy problem from the talmud. Journal of Economic Theory, 36:195-213.

[3] Brânzei, R., Solymosi, T., and Tijs, S. (2005). Strongly essential coalitions and the nucleolus of peer group games. International Journal of Game Theory, 33:447460.

[4] Chardaire, P. (2001). The core and nucleolus of games: A note on a paper by goethe-lundgren et al. Mathematical Programming, 90(1).

[5] Deng, X., Fang, Q., and Sun, X. (2009). Finding nucleolus of flow game. Journal of Combinatorial Optimization, $18(1): 64-86$

[6] Elkind, E., Goldberg, L. A., Goldberg, P., and Wooldridge, M. (2009). Computational complexity of weighted voting games. Annals of Mathematics and Artificial Intelligence, 56:109-131.

[7] Faigle, U., Kern, W., and Kuipers, J. (1998). Computing the nucleolus of min-cost spanning tree games is np-hard. International Journal of Game Theory, 27.

[8] Fromen, B. (1997). Reducing the number of linear programs needed for solving th nucleolus problem of $n$ person game. European Journal of Operntional Research, 98:626-636.

[9] Gillies, D. (1959). Solutions to general non-zero-sum games. In Tucker, A. and Luce, R. E., editors, Contributions to the Theory of Games IV., volume 40 of Ann. Math. Stud., pages 47-85. Princeton University Press.

[10] Gonzalez, S. and Grabisch, M. (2015). Autonomous coalitions. Annals of Operations Research, 235(1):301317.

[11] Göthe-Lundgren, M., Jörnsten, K., and Värbrand, P. (1996). On the nucleolus of the basic vehicle routing game. Mathematical Programming, 72:83-100.

[12] Granot, D., Granot, F., and Zhu, W. R. (1998). Characterization sets for the nucleolus. International Journal of Game Theory, 27(3):359-374.

[13] Greco, G., Malizia, E., Palopoli, L., and Scarcello, F. (2015). Complexity of the nucleolus in compact games.
ACM Transactions on Computation Theory, 7(3).

[14] Groote Schaarsberg, M., Borm, P., Hamers, H., and Reijnierse, H. (2013). Game theoretic analysis of maximum cooperative purchasing situations. Naval Research Logistics, 60:607-624.

[15] Huberman, G. (1980). The nucleolus and essential coalitions. In Bensoussan, A. and Lions, J. L., editors, Analysis and Optimization of Systems, volume 28 of Lecture Notes in Control and Information Sciences, pages 416-422. Elsevier B.V.

[16] Kern, W. and Paulusma, D. (2003). Matching games: the least core and the nucleolus. Mathematics of Operations Research, 28(2):294-308.

[17] Kohlberg, E. (1971). On the nucleolus of a characteristic function game. SIAM J. Appl. Math., 20:62-65.

[18] Kuipers, J. (1996). A polynomial time algorithm for computing the nucleolus of convex games. Report $\mathrm{m} 96-$ 12, University of Maastricht.

[19] Littlechild, S. C. and Owen, G. (1973). A simple expression for the shapley value in a special case. Management Science, 20(3):370-372.

[20] Maschler, M., Peleg, B., and Shapley, L. (1979). Geometric properties of the kernel, nucleolus and related solution concepts. Math. Oper. Res., 4:303-338.

[21] Maschler, M., Potters, J., and Reijnierse, H. (2010). The nucleolus of a standard tree game revisited: a study of its monotonicity and computational properties. International Journal of Game Theory, 39(1-2):89-104.

[22] Maschler, M., Potters, J., and Tijs, S. (1992). The general nucleolus and the reduced game property. International Journal of Game Theory, 21:85-106.

[23] Megiddo, N. (1974). Nucleoluses of compound simple games. SIAM J. Appl. Math., 26(3).

[24] Oishi, T. and Nakayama, M. (2009). Anti-Dual Of Economic Coalitional TU Games. The Japanese Economic Review, 60(4):560-566.

[25] Potters, J. A. M., Reijnierse, H., and Ansing, M. (1996). Computing the nucleolus by solving a prolonged simplex algorithm. Mathematics of Operations Research, 21(3):757-768.

[26] Puerto, J. and Perea, F. (2013). Finding the nucleolus of any $n$-person cooperative game by a single linear program. Computers \& OperationsResearch, 40:2308-2313.

[27] Reijnierse, H. and Potters, J. A. M. (1998). The $\mathcal{B}-$ nucleolus of tu-games. Games and Economic Behavior, 24:77-96.

[28] Sankaran, J. K. (1991). On finding the nucleolus of an $n$-person cooperative game. International Journal of Game Theory, 19:329-338.

[29] Schmeidler, D. (1969). The nucleolus of a characteristic function game. SIAM J. Appl. Math., 17:1163-1170.

[30] Shellshear, E. and Sudhölter, P. (2009). On core stability, vital coalitions, and extendability. Games and Economic Behavior, 67(2):633-644.

[31] Solymosi, T. and Raghavan, T. E. S. (1994). An algorithm for finding the nucleolus of assignment games. International Journal of Game Theory, 23:119-143. 
[32] Solymosi, T. and Sziklai, B. (2015). Universal characterization sets for the nucleolus in balanced games. IEHAS Discussion Papers MT-DP 2015/12.

[33] Sziklai, B. (2015). On the Computation of the $\mathrm{Nu}$ cleolus of Cooperative Transferable Utility Games. Phd thesis, Eötös Loránd University, Budapest. 